\title{
Robust Stability Analysis of Neutral-Type Hybrid Bidirectional Associative Memory Neural Networks with Time-Varying Delays
}

\author{
Wei Feng, ${ }^{1,2}$ Simon X. Yang, ${ }^{1,3}$ and Haixia $W^{1,2}$ \\ ${ }^{1}$ College of Automation, Chongqing University, Chongqing 400044, China \\ ${ }^{2}$ Department of Mathematics and Information Engineering, Chongqing University of Education, Chongqing 400065, China \\ ${ }^{3}$ School of Engineering, University of Guelph, Guelph, ON, Canada N1G 2 W1 \\ Correspondence should be addressed to Wei Feng; fengwit@gmail.com
}

Received 5 March 2014; Accepted 2 May 2014; Published 27 May 2014

Academic Editor: Gani Stamov

Copyright (C) 2014 Wei Feng et al. This is an open access article distributed under the Creative Commons Attribution License, which permits unrestricted use, distribution, and reproduction in any medium, provided the original work is properly cited.

\begin{abstract}
The global asymptotic robust stability of equilibrium is considered for neutral-type hybrid bidirectional associative memory neural networks with time-varying delays and parameters uncertainties. The results we obtained in this paper are delay-derivativedependent and establish various relationships between the network parameters only. Therefore, the results of this paper are applicable to a larger class of neural networks and can be easily verified when compared with the previously reported literature results. Two numerical examples are illustrated to verify our results.
\end{abstract}

\section{Introduction}

Stability analysis of neural networks is an issue of both theoretical and practical importance due to the fact that in some applications the designed neural network is required to have a unique and stable equilibrium point [1-3]. Time delays are unavoidably encountered in the implementation of neural networks, which may cause undesirable dynamic network behaviors such as oscillation and instability. On the other hand, in practice, the weight coefficients of the neurons depend on certain resistance and capacitance values which are subject to uncertainties. In the design of neural networks, it is important to ensure that the system is stable with respect to these uncertainties.

It is well known that a series of neural networks related to bidirectional associative memory (BAM) models have been proposed by Kosko $[4,5]$. These models generalized the single-layer autoassociative Hebbian correlation to a twolayer pattern-matched heteroassociative circuit. This class of networks has been successfully applied to pattern recognition and artificial intelligence. A great number of results for BAM neural networks concerning the existence of equilibrium point and global asymptotic or robust stability have been derived [6-32].
Moreover, due to the complicated dynamic properties of the neural cells in the real world, the existing neural network models in many cases cannot characterize the properties of a neural reaction process precisely. It is natural and important that systems will contain some information about the derivative of the past state to further describe and model the dynamics for such complex neural reactions [33, 34]. However, the stability analysis of BAM neural networks of neutral type has been investigated by only a few researchers [18, 35-37].

However, the existing stability results $[18,36,37]$ derived for the BAM neural networks can be applicable when only a pure delayed neural network model is considered. Recently, a more general class of BAM neural network models, called the hybrid BAM neural network in which both instantaneous and delayed signaling occur, was considered and some sufficient condition for robust stability of this class of BAM neural networks has been presented $[23,25,38]$. But, up to now, there are few results on stability of neutral-type hybrid BAM neural networks with time-varying delays.

Motivated by the preceding discussion, in this paper, we are going to deal with the problem of global asymptotic robust stability for neutral-type hybrid bidirectional associative memory neural networks with time-varying delays 
and parameters uncertainties. By constructing a novel Lyapunov functional, novel delay-derivative-dependent criteria are derived. Finally, two examples are provided to demonstrate the effectiveness of the obtained results.

Throughout this paper, we will use the following notations: let $v=\left(v_{1}, v_{2}, \ldots, v_{n}\right)^{T} \in \mathfrak{R}^{n}$ be a column vector and let $Q=\left(q_{i j}\right)_{n \times n}$ be a real matrix. The three commonly used vector norms $\|v\|_{1},\|v\|_{2}$, and $\|v\|_{\infty}$ are defined as

$$
\begin{gathered}
\|v\|_{1}=\sum_{i=1}^{n}\left|v_{i}\right|, \quad\|v\|_{2}=\sqrt{\sum_{i=1}^{n}\left|v_{i}^{2}\right|}, \\
\|v\|_{\infty}=\max _{1 \leq i \leq n}\left|v_{i}\right| .
\end{gathered}
$$

The three commonly used matrix norms $\|Q\|_{1},\|Q\|_{2}$, and $\|Q\|_{\infty}$ are defined as follows:

$$
\begin{gathered}
\|Q\|_{1}=\max _{1 \leq j \leq n} \sum_{i=1}^{n}\left|q_{i j}\right|, \quad\|Q\|_{2}=\left[\lambda_{M}\left(Q^{T} Q\right)\right]^{1 / 2}, \\
\|Q\|_{\infty}=\max _{1 \leq i \leq n} \sum_{j=1}^{n}\left|q_{i j}\right| .
\end{gathered}
$$

For the vector $v=\left(v_{1}, v_{2}, \ldots, v_{n}\right)^{T},|v|$ will denote $v=$ $\left(\left|v_{1}\right|,\left|v_{2}\right|, \ldots,\left|v_{n}\right|\right)^{T}$. For the matrix $Q=\left(q_{i j}\right)_{n \times n}$, the matrix $|Q|$ will denote $|Q|=\left(\left|q_{i j}\right|\right)_{n \times n}$ and $\lambda_{m}(Q)$ and $\lambda_{M}(Q)$ will denote the minimum and maximum eigenvalues of $Q$, respectively. If $P=\left(p_{i j}\right)_{n \times n}$ and $Q=\left(q_{i j}\right)_{n \times n}$ are two real symmetric matrices, then $P \leq Q$ will imply that $p_{i j} \leq q_{i j}$, $i, j=1,2, \ldots, n$.

\section{Problem Formulation}

Dynamical behavior of a neutral-type hybrid BAM neural network with time-varying delays is described by the following set of differential equations:

$$
\begin{aligned}
\dot{u}_{i}(t)+ & \sum_{j=1}^{m} e_{j i} \dot{u}_{i}(t-h) \\
= & -a_{i} u_{i}(t)+\sum_{j=1}^{m} w_{j i} \widetilde{f}_{j}\left(z_{j}(t)\right) \\
& +\sum_{j=1}^{m} w_{j i}^{\tau} \tilde{f}_{j}\left(z_{j}(t-\tau(t))\right)+I_{i}, \quad i=1,2, \ldots, n, n, \quad j=1,2, \ldots, m, \\
\dot{z}_{j}(t)+ & \sum_{i=1}^{n} \varepsilon_{i j} \dot{z}_{j}(t-d) \\
= & -b_{j} z_{j}(t)+\sum_{i=1}^{n} v_{i j} \widetilde{g}_{i}\left(u_{i}(t)\right) \\
& +\sum_{i=1}^{n} v_{i j}^{\sigma} \widetilde{g}_{i}\left(u_{i}(t-\sigma(t))\right)+J_{j}, \quad j=1
\end{aligned}
$$

in which $u=\left(u_{1}, u_{2}, \ldots, u_{n}\right)^{T}$ and $z=\left(z_{1}, z_{2}, \ldots, z_{m}\right)^{T}$ are the neuron state vectors, $a_{i}$ and $b_{j}$ denote the neuron charging time constants and passive decay rates, respectively, $w_{j i}, w_{j i}^{\tau}, v_{i j}$, and $v_{i j}^{\sigma}$ are the connection weights at the time $t$, $\tilde{f}_{i}$ and $\tilde{g}_{j}$ represent the activation functions of the neurons and the propagational signal functions, respectively, and $I_{i}$, $J_{j}$, denote the external inputs. $h>0$ and $d>0$ are positive constants which correspond to the finite speed of axonal signal transmission.

It will be assumed that $a_{i}, b_{j}, w_{j i}, w_{j i}^{\tau}, v_{i j}, v_{i j}^{\sigma}, e_{j i}$, and $\varepsilon_{i j}$ in system (3) are uncertain but bounded and belong to the following intervals:

$$
\begin{aligned}
& A_{I}:=\left\{A=\operatorname{diag}\left(a_{i}\right): 0<\underline{A} \leq A \leq \bar{A}\right. \text {, i.e., } \\
& \left.0<\underline{a}_{i} \leq a_{i} \leq \bar{a}_{i}, i=1,2, \ldots, n, \forall A \in A_{I}\right\}, \\
& B_{I}:=\left\{B=\operatorname{diag}\left(b_{j}\right): 0<\underline{B} \leq B \leq \bar{B}\right. \text {, i.e., } \\
& \left.0<\underline{b}_{j} \leq b_{j} \leq \bar{b}_{j}, j=1,2, \ldots, m, \forall B \in B_{I}\right\}, \\
& W_{I}:=\left\{W=\left(w_{j i}\right)_{m \times n}: \underline{W} \leq W \leq \bar{W} \text {, i.e., } \underline{w}_{j i} \leq w_{j i} \leq \bar{w}_{j i}\right. \text {, } \\
& \left.i=1,2, \ldots, n ; j=1,2, \ldots, m, \forall W \in W_{I}\right\}, \\
& V_{I}:=\left\{V=\left(v_{i j}\right)_{n \times m}: \underline{V} \leq V \leq \bar{V}\right. \text {, i.e., } \\
& \underline{v}_{i j} \leq v_{i j} \leq \bar{v}_{i j}, i=1,2, \ldots, n \text {; } \\
& \left.j=1,2, \ldots, m, \forall V \in V_{I}\right\}, \\
& W_{I}^{\tau}:=\left\{W^{\tau}=\left(w_{j i}^{\tau}\right)_{m \times n}: \underline{W}^{\tau} \leq W \leq \bar{W}^{\tau}\right. \text {, i.e., } \\
& \underline{w}_{j i}^{\tau} \leq w_{j i}^{\tau} \leq \bar{w}_{j i}^{\tau}, i=1,2, \ldots, n ; \\
& \left.j=1,2, \ldots, m, \forall W^{\tau} \in W_{I}^{\tau}\right\}, \\
& V_{I}^{\sigma}:=\left\{V^{\sigma}=\left(v_{i j}^{\sigma}\right)_{n \times m}: \underline{V}^{\sigma} \leq V \leq \bar{V}^{\sigma}\right. \text {, i.e., } \\
& \underline{v}_{i j}^{\sigma} \leq v_{i j}^{\sigma} \leq \bar{v}_{i j}^{\sigma}, i=1,2, \ldots, n ; \\
& \left.j=1,2, \ldots, m, \forall V^{\sigma} \in V_{I}^{\sigma}\right\}, \\
& E_{I}:=\left\{E=\left(e_{j i}\right)_{m \times n}: \underline{E} \leq E \leq \bar{E} \text {, i.e., } \underline{e}_{j i} \leq e_{j i} \leq \bar{e}_{j i},\right. \\
& \left.i=1,2, \ldots, n ; j=1,2, \ldots, m, \forall E \in E_{I}\right\}, \\
& \Sigma_{I}:=\left\{\Sigma=\left(\varepsilon_{i j}\right)_{n \times m}: \underline{\Sigma} \leq \Sigma \leq \bar{\Sigma} \text {, i.e., } \underline{\varepsilon}_{i j} \leq \varepsilon_{i j} \leq \bar{\varepsilon}_{i j},\right. \\
& \left.i=1,2, \ldots, n ; j=1,2, \ldots, m, \forall \Sigma \in \Sigma_{I}\right\} .
\end{aligned}
$$

(H1) $\tau(t) \geq 0$ and $\sigma(t) \geq 0$ are differentiable functions that satisfy

$$
0 \leq \tau(t) \leq \bar{\tau}, \quad 0 \leq \sigma(t) \leq \bar{\sigma},
$$




$$
\dot{\tau}(t) \leq \mu_{1} \leq 1, \quad \dot{\sigma}(t) \leq \mu_{2} \leq 1
$$

for all $t \geq 0$ and prescribed scalars $\bar{\tau}>0, \bar{\sigma}>0$, $\mu_{1}>0$, and $\mu_{2}>0$.

The activation functions satisfy the following properties.

(H2) There exist some positive constants $\ell_{i}, i=1,2, \ldots, n$, and $\kappa_{j}, j=1,2, \ldots, m$, such that

$$
0 \leq \frac{\tilde{f}_{i}(\bar{x})-\tilde{f}_{i}(\bar{y})}{\bar{x}-\bar{y}} \leq \ell_{i}, \quad 0 \leq \frac{\tilde{g}_{j}(\widehat{x})-\tilde{g}_{j}(\widehat{y})}{\widehat{x}-\widehat{y}} \leq \kappa_{j}
$$

for all $\bar{x}, \bar{y}, \widehat{x}, \hat{y} \in \mathfrak{R}$.

(H3) There exist positive constants $M_{i}, i=1,2, \ldots, n$, and $L_{j}, j=1,2, \ldots, m$, such that $\left|\widetilde{g}_{i}(u)\right| \leq M_{i}$ and $\left|\widetilde{\mathfrak{g}}_{j}(z)\right| \leq L_{i}$ for all $u, z \in \Re$. Note that this assumption implies that the activation functions are bounded.

Assume that $u^{*}=\left(u_{1}^{*}, u_{2}^{*}, \ldots, u_{n}^{*}\right)^{T}$ and $z^{*}=\left(z_{1}^{*}, z_{2}^{*}, \ldots\right.$ ,$\left.z_{m}^{*}\right)^{T}$ are the equilibrium points of the system. In order to simplify our analysis, we transform the equilibrium points to the origin by the relationship

$$
x_{i}(t)=u_{i}(t)-u_{i}^{*}, \quad y_{i}(t)=z_{j}(t)-z_{j}^{*} .
$$

Then, the transformed system is as follows:

$$
\begin{array}{r}
\dot{x}(t)+E \dot{x}(t-h) \\
=-A x(t)+W f(y(t))+W^{\tau} f(y(t-\tau(t))), \\
i=1,2, \ldots, n, \\
\dot{y}(t)+\Sigma \dot{y}(t-d) \\
=-B y(t)+V g(x(t))+V^{\sigma} g(x(t-\sigma(t))), \\
j=1,2, \ldots, m,
\end{array}
$$

where $x(t)=\left(x_{1}(t), x_{2}(t), \ldots, x_{n}(t)\right)^{T}, y(t)=\left(y_{1}(t)\right.$, $\left.y_{2}(t), \ldots, y_{n}(t)\right)^{T}, g(x(t))=\left(g_{1}\left(x_{1}(t)\right), g_{2}\left(x_{2}(t)\right), \ldots, g_{n}\left(x_{n}\right.\right.$ $(t)))^{T}, f(y(t))=\left(f_{1}\left(y_{1}(t)\right), f_{2}\left(y_{2}(t)\right), \ldots, f_{n}\left(y_{n}(t)\right)\right)^{T}, g(x(t-$ $\sigma(t)))=\left(g_{1}\left(x_{1}(t-\sigma(t)), g_{2}\left(x_{2}(t-\sigma(t))\right), \ldots, g_{n}\left(x_{n}(t-\right.\right.\right.$ $\sigma(t))))^{T}, f(y(t-\tau))=\left(f_{1}\left(y_{1}(t-\tau(t))\right), f_{2}\left(y_{2}(t-\right.\right.$ $\left.\tau(t))), \ldots, f_{m}\left(y_{m}(t-\tau(t))\right)\right)^{T}$. The functions $g_{i}\left(x_{i}\right)$, and $f_{j}\left(y_{j}\right)$ are of the form

$$
\begin{gathered}
g_{i}\left(x_{i}(\cdot)\right)=\tilde{g}_{i}\left(x_{i}(\cdot)+u_{i}^{*}\right)-\tilde{g}_{i}\left(u_{i}^{*}\right), \quad i=1,2, \ldots, n, \\
f\left(y_{j}(\cdot)\right)=\tilde{f}_{j}\left(y_{j}(\cdot)+z_{j}^{*}\right)-\tilde{f}_{j}\left(z_{j}^{*}\right), \quad j=1,2, \ldots, m .
\end{gathered}
$$

It can be verified that the functions $g_{i}$ and $f_{j}$ satisfy the assumptions on $g_{i}$ and $f_{j}$; that is, $g_{i} \in \beta, f_{j} \in \kappa$, and $g_{i} \in \beta$, $f_{j} \in \beta$ implies that $g_{i} \in \beta$ and $f_{j} \in \beta$, respectively. We also note that $g_{i}(0)=0$ and $f_{j}(0)=0, i=1,2, \ldots, n$.

By assumption (H2) and the above equations, we can have

$$
0 \leq \frac{f(y)}{y} \leq \ell_{i}, \quad 0 \leq \frac{g(x)}{x} \leq \kappa_{i} .
$$

\section{Preliminaries}

In this paper, we will assume that the norms of the matrices $A, B, W=\left(W_{j i}\right), W^{\tau}=\left(W_{j i}^{\tau}\right), V=\left(V_{i j}\right)$, and $V^{\sigma}=\left(W_{i j}^{\sigma}\right)$ are bounded. Based on this property, we can directly observe the following facts.

Fact 1. If $A, B, W=\left(W_{j i}\right), W^{\tau}=\left(W_{j i}^{\tau}\right), V=\left(V_{i j}\right)$, and $V^{\sigma}=$ $\left(W_{i j}^{\sigma}\right)$ satisfy the parameter ranges defined by (4) and have bounded norms, then there exist some positive constants $\sigma(W), \sigma\left(W^{\tau}\right), \sigma(V)$, and $\sigma\left(V^{\sigma}\right)$ :

$$
\begin{gathered}
\|A\|_{2} \leq \sigma(A), \quad\|B\|_{2} \leq \sigma(B), \quad\|W\|_{2} \leq \sigma(W), \\
\left\|W^{\tau}\right\|_{2} \leq \sigma\left(W^{\tau}\right), \quad\|V\|_{2} \leq \sigma(V), \\
\left\|V^{\sigma}\right\|_{2} \leq \sigma\left(V^{\sigma}\right) .
\end{gathered}
$$

Lemma 1 (Faydasicok and Arik [39]). For $W \in W_{I}:=\{W=$ $\left(w_{i j}\right): \underline{W} \leq W \leq \bar{W}$, i.e., $\left.\underline{w}_{i j} \leq w_{i j} \leq \bar{w}_{i j}, i, j=1,2, \ldots, n\right\}$, the following equation holds:

$$
\sigma_{1}(W)=\sqrt{\left\|\left|W^{* T} W^{*}\right|+2\left|W^{* T}\right| W_{*}+W_{*}^{T} W_{*}\right\|_{2}},
$$

where $W^{*}=(1 / 2)(\bar{W}+\underline{W})$ and $W_{*}=(1 / 2)(\bar{W}-\underline{W})$.

Lemma 2 (Cao et al. [40]). For $W \in W_{I}:=\left\{W=\left(w_{i j}\right): \underline{W} \leq\right.$ $W \leq \bar{W}$, i.e., $\left.\underline{w}_{i j} \leq w_{i j} \leq \bar{w}_{i j}, i, j=1,2, \ldots, n\right\}$, the following equation holds:

$$
\sigma_{2}(W)=\left\|W^{*}\right\|_{2}+\left\|W_{*}\right\|_{2}
$$

where $W^{*}=(1 / 2)(\bar{W}+\underline{W})$ and $W_{*}=(1 / 2)(\bar{W}-\underline{W})$.

Lemma 3 (Ensari and Arik [41]). For $W \in W_{I}:=\{W=$ $\left(w_{i j}\right): \underline{W} \leq W \leq \bar{W}$, i.e., $\left.\underline{w}_{i j} \leq w_{i j} \leq \bar{w}_{i j}, i, j=1,2, \ldots, n\right\}$, the following equation holds:

$$
\sigma_{3}(W)=\sqrt{\left\|W^{*}\right\|_{2}^{2}+\left\|W_{*}\right\|_{2}^{2}+2\left\|W_{*}^{T}\left|W^{*}\right|\right\|_{2}},
$$

where $W^{*}=(1 / 2)(\bar{W}+\underline{W})$ and $W_{*}=(1 / 2)(\bar{W}-\underline{W})$.

Lemma 4 (Singh [42]). For $W \in W_{I}:=\left\{W=\left(w_{i j}\right): \underline{W} \leq\right.$ $W \leq \bar{W}$, that $\left.i s, \underline{w}_{i j} \leq w_{i j} \leq \bar{w}_{i j}, i, j=1,2, \ldots, n\right\}$, the following equation holds:

$$
\sigma_{4}(W)=\|\widehat{W}\|_{2}
$$

where $\widehat{W}=\left(\widehat{w}_{i j}\right)_{n \times n}$ with $\widehat{w}_{i j}=\max \left\{\left|\underline{w}_{i j}\right|,\left|\bar{w}_{i j}\right|\right\}$.

Lemma 5. For any two vectors $\omega=\left(\omega_{1}, \omega_{2}, \ldots, \omega_{n}\right)^{T}$ and $v=$ $\left(v_{1}, v_{2}, \ldots, v_{n}\right)^{T}$, the following inequality holds:

$$
2 \omega^{T} v=2 v^{T} \omega \leq \gamma \omega^{T} \omega+\frac{1}{\gamma} v^{T} v
$$

where $\gamma$ is any positive constant. 


\section{Global Robust Stability Results}

Note that the equilibrium point of system (3) is globally asymptotically stable, if the origin of system (8) is a globally asymptotically stable equilibrium point. Therefore, in order to prove the global asymptotic stability of the equilibrium point of system (3), it will be sufficient to prove the global asymptotic stability of the origin of system (8). We can now proceed with the following result.

Theorem 6. For given scalars $0 \leq \mu_{1} \leq 1$ and $0 \leq \mu_{2} \leq 1$, let the activation functions satisfy assumptions (H2) and (H3) and let the network parameters satisfy (4). Then, the origin of neural network model (8) is globally asymptotically stable, if there exist positive diagonal matrices $H_{1}=\operatorname{diag}\left(h_{1 i}>0\right)$ and $\mathrm{H}_{2}=\operatorname{diag}\left(h_{2 j}>0\right)$, positive definite matrices $R$, and $T$, and four positive scalars $\alpha, \beta, \chi$, and $\delta$, such that

$$
\begin{gathered}
\theta_{1}=\|\underline{A}\|_{2}-\left\|H_{1}\right\|_{2}-\frac{1}{\alpha}-\frac{1}{\beta\left(1-\mu_{1}\right)}-\sigma^{2}(A)\left\|R^{-1}\right\|_{2}>0, \\
\theta_{2}=\left\|H_{1}\right\|_{2}-3 \sigma^{2}(E)\|R\|_{2}>0, \\
\theta_{3}=\|\underline{A}\|_{2}\left\|K^{-2}\right\|_{2}-\chi \sigma^{2}(V)-\sigma^{2}(V)\left\|T^{-1}\right\|_{2} \\
-\frac{1}{\left(1-\mu_{2}\right)} \sigma^{2}\left(V^{\sigma}\right)\left\|T^{-1}\right\|_{2}-\delta \sigma^{2}\left(V^{\sigma}\right)>0, \\
\theta_{4}=\left\|\underline{B}_{2}-\right\| H_{2}\left\|_{2}-\frac{1}{\chi}-\frac{1}{\delta\left(1-\mu_{2}\right)}-\sigma^{2}(B)\right\| T^{-1} \|_{2}>0, \\
\theta_{5}=\left\|H_{2}\right\|_{2}-3 \sigma^{2}(\Sigma)\|T\|_{2}>0, \\
\theta_{6}=\|\underline{B}\|_{2}\left\|L^{-2}\right\|_{2}-\alpha \sigma^{2}(W)-\sigma^{2}(W)\left\|R^{-1}\right\|_{2} \\
-\frac{1}{\left(1-\mu_{1}\right)} \sigma^{2}\left(W^{\tau}\right)\left\|R^{-1}\right\|_{2}-\beta \sigma^{2}\left(W^{\tau}\right)>0,
\end{gathered}
$$

where

$$
\begin{gathered}
\sigma(A)=\min \left\{\sqrt{\left\|\left|A^{* T} A^{*}\right|+2\left|A^{* T}\right| A_{*}+A_{*}^{T} A_{*}\right\|_{2}},\right. \\
\left\|A^{*}\right\|_{2}+\left\|A_{*}\right\|_{2}, \\
\left.\sqrt{\left\|A^{*}\right\|_{2}^{2}+\left\|A_{*}\right\|_{2}^{2}+2\left\|A_{*}^{T}\left|A^{*}\right|\right\|_{2}, \|} \mid \widehat{A} \|_{2}\right\}, \\
\sigma(B)=\min \left\{\sqrt{\left\|B^{* T} B^{*}|+2| B^{* T} \mid B_{*}+B_{*}^{T} B_{*}\right\|_{2}},\right. \\
\left\|B^{*}\right\|_{2}+\left\|B_{*}\right\|_{2}, \\
\sigma(W)=\min \left\{\sqrt{\left\|B^{*}\right\|_{2}^{2}+\left\|B_{*}\right\|_{2}^{2}+2\left\|B_{*}^{T}\left|B^{*}\right|\right\|_{2}},\|\widehat{B}\|_{2}\right\}, \\
\left\|W^{*}\right\|_{2}+\left\|W_{*}\right\|_{2}, \\
\left.\sqrt{\left\|W^{*}\right\|_{2}^{2}+\left\|W_{*}\right\|_{2}^{2}+2\left\|W_{*}+W_{*}^{T}\left|W^{*}\right|\right\|_{2}},\|\widehat{W}\|_{2}\right\},
\end{gathered}
$$

$\sigma(V)$

$$
=\min \left\{\sqrt{\left\|\left|V^{* T} V^{*}\right|+2\left|V^{* T}\right| V_{*}+V_{*}^{T} V_{*}\right\|_{2}},\right.
$$$$
\left\|V^{*}\right\|_{2}+\left\|V_{*}\right\|_{2}
$$$$
\left.\sqrt{\left\|V^{*}\right\|_{2}^{2}+\left\|V_{*}\right\|_{2}^{2}+2\left\|V_{*}^{T}\left|V^{*}\right|\right\|_{2}},\|\widehat{V}\|_{2}\right\},
$$

$\sigma\left(W^{\tau}\right)$

$$
\begin{aligned}
=\min \{ & \sqrt{\left\|\left|W^{\tau * T} W^{\tau *}\right|+2\left|W^{\tau * T}\right| W_{*}^{\tau}+W_{*}^{\tau T} W_{*}^{\tau}\right\|_{2},} \\
& \left\|W^{\tau *}\right\|_{2}+\left\|W_{*}^{\tau}\right\|_{2}, \\
& \left.\sqrt{\left\|W^{\tau *}\right\|_{2}^{2}+\left\|W_{*}^{\tau}\right\|_{2}^{2}+2\left\|W_{*}^{\tau T}\left|W^{\tau *}\right|\right\|_{2}},\left\|\widehat{W}^{\tau}\right\|_{2}\right\},
\end{aligned}
$$

$\sigma\left(V^{\sigma}\right)$

$$
=\min \left\{\sqrt{\left\|\left|V^{\sigma * T} V^{\sigma *}\right|+2\left|V^{\sigma * T}\right| V_{*}^{\sigma}+V_{*}^{\sigma T} V_{*}^{\sigma}\right\|_{2}},\right.
$$$$
\left\|V^{\sigma *}\right\|_{2}+\left\|V_{*}^{\sigma}\right\|_{2} \text {, }
$$$$
\left.\sqrt{\left\|V^{\sigma *}\right\|_{2}^{2}+\left\|V_{*}^{\sigma}\right\|_{2}^{2}+2\left\|V_{*}^{\sigma T}\left|V^{\sigma *}\right|\right\|_{2}},\left\|\widehat{V}^{\sigma}\right\|_{2}\right\},
$$$$
A^{*}=\frac{1}{2}(\bar{A}+\underline{A}), \quad A_{*}=\frac{1}{2}(\bar{A}-\underline{A}),
$$$$
B^{*}=\frac{1}{2}(\bar{B}+\underline{B}), \quad B_{*}=\frac{1}{2}(\bar{B}-\underline{B}),
$$$$
W^{*}=\frac{1}{2}(\bar{W}+\underline{W}), \quad W_{*}=\frac{1}{2}(\bar{W}-\underline{W}),
$$$$
W^{\tau *}=\frac{1}{2}\left(\bar{W}^{\tau}+\underline{W}^{\tau}\right), \quad W_{*}^{\tau}=\frac{1}{2}\left(\bar{W}^{\tau}-\underline{W}^{\tau}\right),
$$$$
V^{*}=\frac{1}{2}(\bar{V}+\underline{V}), \quad V_{*}=\frac{1}{2}(\bar{V}-\underline{V}),
$$$$
V^{\sigma *}=\frac{1}{2}\left(\bar{V}^{\sigma}+\underline{V}^{\sigma}\right), \quad V_{*}^{\sigma}=\frac{1}{2}\left(\bar{V}^{\sigma}-\underline{V}^{\sigma}\right),
$$

$$
\widehat{A}=\operatorname{diag}\left(\widehat{a}_{i}\right) \quad \text { with } \widehat{a}_{i}=\max \left\{\left|\underline{a}_{i}\right|,\left|\bar{a}_{i}\right|\right\},
$$$$
\widehat{B}=\operatorname{diag}\left(\widehat{b}_{j}\right) \text { with } \widehat{b}_{j}=\max \left\{\left|\underline{b}_{j}\right|,\left|\bar{b}_{j}\right|\right\} \text {, }
$$$$
\widehat{W}=\left(\widehat{w}_{i j}\right)_{n \times n} \text { with } \widehat{w}_{i j}=\max \left\{\left|\underline{w}_{i j}\right|,\left|\bar{w}_{i j}\right|\right\} \text {, }
$$$$
\widehat{W}^{\tau}=\left(\widehat{w}_{i j}^{\tau}\right)_{n \times n} \text { with } \widehat{w}_{i j}^{\tau}=\max \left\{\left|\underline{w}_{i j}^{\tau}\right|,\left|\bar{w}_{i j}^{\tau}\right|\right\} \text {, }
$$

$$
K=\operatorname{diag}\left(\kappa_{j}>0\right),
$$

$\widehat{V}=\left(\widehat{v}_{i j}\right)_{n \times n}$ with $\widehat{v}_{i j}=\max \left\{\left|\underline{v}_{i j}\right|,\left|\bar{v}_{i j}\right|\right\}$,

$\widehat{V}^{\sigma}=\left(\widehat{v}_{i j}^{\sigma}\right)_{n \times n}$ with $\widehat{v}_{i j}^{\sigma}=\max \left\{\left|\underline{v}_{i j}^{\sigma}\right|,\left|\bar{v}_{i j}^{\sigma}\right|\right\}$,

$$
L=\operatorname{diag}\left(\ell_{i}>0\right) .
$$


Proof. Define the following positive definite Lyapunov functional:

$$
\begin{aligned}
V(x( & t), y(t)) \\
= & {[x(t)+E x(t-h)]^{T}[x(t)+E x(t-h)] } \\
& +\sum_{i=1}^{n} h_{1 i} \int_{t-h}^{t} x_{i}^{2}(s) d s \\
& +[y(t)+\Sigma y(t-d)]^{T}[y(t)+\Sigma y(t-d)] \\
& +\sum_{j=1}^{m} h_{2 j} \int_{t-d}^{t} y_{j}^{2}(s) d s \\
& +\left(\gamma_{1}+\beta_{1}\right) \sum_{j=1}^{m} \int_{t-\tau(t)}^{t} f_{j}\left(y_{j}(\xi)\right) d \xi \\
& +\left(\gamma_{2}+\beta_{2}\right) \sum_{i=1}^{n} \int_{t-\sigma(t)}^{t} g_{i}\left(x_{i}(\xi)\right) d \xi .
\end{aligned}
$$

The derivative of $V(x(t), y(t))$ along the trajectories of the system is obtained as follows:

$$
\begin{aligned}
\dot{V}(x( & (t), y(t)) \\
= & -2 x^{T}(t) A x(t)+2 x^{T}(t) W f(y(t)) \\
& +2 x^{T}(t) W^{\tau} f(y(t-\tau(t))) \\
& -2 x^{T}(t-h) E^{T} A x(t)+2 x^{T}(t-h) E^{T} W f(y(t)) \\
& +2 x^{T}(t-h) E^{T} W^{\tau} f(y(t-\tau(t))) \\
& +x^{T}(t) H_{1} x(t)-x^{T}(t-h) H_{1} x(t-h) \\
& -2 y^{T}(t) B y(t)+2 y^{T}(t) V g(x(t)) \\
& +2 y^{T}(t) V^{\sigma} g(x(t-\sigma(t))) \\
& -2 y^{T}(t-d) \Sigma^{T} B y(t)+2 y^{T}(t-d) \Sigma^{T} V g(x(t)) \\
& +2 y^{T}(t-d) \Sigma^{T} V^{\sigma} g(x(t-\sigma(t))) \\
& +y^{T}(t) H_{2} y(t)-y^{T}(t-d) H_{2} y(t-d) \\
& +\gamma_{1}\|f(y(t))\|_{2}^{2}-\gamma_{1}\left(1-\mu_{1}\right)\|f(y(t-\tau(t)))\|_{2}^{2} \\
& +\beta_{1}\|f(y(t))\|_{2}^{2}-\beta_{1}\left(1-\mu_{1}\right)\|f(y(t-\tau(t)))\|_{2}^{2} \\
& +\gamma_{2}\|g(x(t))\|_{2}^{2}-\gamma_{2}\left(1-\mu_{2}\right)\|g(x(t-\sigma(t)))\|_{2}^{2} \\
& \left.+\beta_{2}(t)\right)\left\|_{2}^{2}-\beta_{2}\left(1-\mu_{2}\right)\right\| g(x(t-\sigma(t))) \|_{2}^{2} .
\end{aligned}
$$

We can write the following inequalities as follows:

$$
\begin{aligned}
& 2 x^{T}(t) W f(y(t)) \\
& \leq \frac{1}{\alpha} x^{T}(t) x(t)+\alpha f^{T}(y(t)) W^{T} W f(y(t)) \\
& \leq \frac{1}{\alpha}\|x(t)\|_{2}^{2}+\alpha\|W\|_{2}^{2}\|f(y(t))\|_{2}^{2}, \\
& 2 x^{T}(t) W^{\tau} f(y(t-\tau)) \\
& \leq \frac{1}{\beta\left(1-\mu_{1}\right)} x^{T}(t) x(t) \\
& +\beta\left(1-\mu_{1}\right) f^{T}(y(t-\tau(t))) W^{\tau T} \\
& \times W^{\tau} f(y(t-\tau(t))) \\
& \leq \frac{1}{\beta\left(1-\mu_{1}\right)}\|x(t)\|_{2}^{2} \\
& +\beta\left(1-\mu_{1}\right)\left\|W^{\tau}\right\|_{2}^{2} \\
& \times\|f(y(t-\tau(t)))\|_{2}^{2}, \\
& -2 x^{T}(t-h) E^{T} A x(t) \\
& \leq x^{T}(t-h) E^{T} R E x(t-h) \\
& +x^{T}(t) A^{T} R^{-1} A x(t) \\
& \leq\|E\|_{2}^{2}\|R\|_{2}\|x(t-h)\|_{2}^{2} \\
& +\|A\|_{2}^{2}\left\|R^{-1}\right\|_{2}\|x(t)\|_{2}^{2} \text {, } \\
& 2 x^{T}(t-h) E^{T} W f(y(t)) \\
& \leq x^{T}(t-h) E^{T} R E x(t-h) \\
& +f(y(t)) W^{T} R^{-1} W f(y(t)) \\
& \leq\|E\|_{2}^{2}\|R\|_{2}\|x(t-h)\|_{2}^{2} \\
& +\|W\|_{2}^{2}\left\|R^{-1}\right\|_{2}\|f(y(t))\|_{2}^{2}, \\
& 2 x^{T}(t-h) E^{T} W^{\tau} f(y(t-\tau)) \\
& \leq x^{T}(t-h) E^{T} R E x(t-h) \\
& +f(y(t-\tau(t))) W^{\tau T} \\
& \times R^{-1} W^{\tau} f(y(t-\tau(t))) \\
& \leq\|E\|_{2}^{2}\|R\|_{2}\|x(t-h)\|_{2}^{2} \\
& +\left\|W^{\tau}\right\|_{2}^{2}\left\|R^{-1}\right\|_{2}\|f(y(t-\tau(t)))\|_{2}^{2}, \\
& 2 y^{T}(t) V g(x(t)) \\
& \leq \frac{1}{\chi} y^{T}(t) y(t)+\chi g^{T}(x(t))
\end{aligned}
$$




$$
\begin{aligned}
& \times V^{T} V g(x(t)) \\
& \leq \frac{1}{\chi}\|y(t)\|_{2}^{2}+\chi\|V\|_{2}^{2}\|g(x(t))\|_{2}^{2}, \\
& 2 y^{T}(t) V^{\sigma} g(x(t-\sigma)) \\
& \leq \frac{1}{\delta\left(1-\mu_{2}\right)} y^{T}(t) y(t)+\delta\left(1-\mu_{2}\right) g^{T} \\
& \times(x(t-\sigma(t))) V^{\sigma T} V^{\sigma} g(x(t-\sigma(t))) \\
& \leq \frac{1}{\delta\left(1-\mu_{2}\right)}\|y(t)\|_{2}^{2} \\
&+\delta\left(1-\mu_{2}\right)\left\|V^{\sigma}\right\|_{2}^{2}\|g(x(t-\sigma(t)))\|_{2}^{2}, \\
&-2 y^{T}(t-d) \Sigma^{T} B y(t) \leq y^{T}(t-d) \Sigma^{T} T \Sigma y(t-d) \\
&+y^{T}(t) B^{T} T^{-1} B y(t) \\
& \leq\|\Sigma\|_{2}^{2}\|T\|_{2}\|y(t-d)\|_{2}^{2} \\
&+\|B\|_{2}^{2}\left\|T^{-1}\right\|\left\|_{2}\right\| y(t) \|_{2}^{2}, \\
& \leq\|\Sigma\|_{2}^{2}\|T\|_{2}\|y(t-d)\|_{2}^{2} \\
&+\left\|V^{\sigma}\right\|_{2}^{2}\left\|T^{-1}\right\|_{2}\|g(x(t-\sigma(t)))\|_{2}^{2} . \\
& \leq y^{T}(t-d) \Sigma^{T} V g(x(t)) \\
& \leq y^{T}(t-d) \Sigma^{T} T \Sigma y(t-d) \\
&+g^{T}(x(t)) V^{T} T^{-1} V g(x(t)) \\
&+\|V\|_{2}^{2}\|T\|_{2}\|y(t-d)\|_{2}^{2} \\
&+g^{T}\left(x\left\|_{2}\right\| g(x(t)) \|_{2}^{2},\right. \\
&
\end{aligned}
$$

Combining (22)-(31) into (21) and considering

$$
\begin{aligned}
& x^{T}(t) x(t) \geq g^{T}(x(t)) K^{-2} g(x(t)), \\
& y^{T}(t) y(t) \geq f^{T}(y(t)) L^{-2} f(y(t)),
\end{aligned}
$$

we have

$$
\begin{aligned}
& \dot{V}(x(t), y(t)) \\
& \leq-\left(\|\underline{A}\|_{2}-\left\|H_{1}\right\|_{2}-\frac{1}{\alpha}-\frac{1}{\beta\left(1-\mu_{1}\right)}-\|A\|_{2}^{2}\left\|R^{-1}\right\|_{2}\right) \\
& \times\|x(t)\|_{2}^{2} \\
& \text { - }\left(\left\|H_{1}\right\|_{2}-3\|E\|_{2}^{2}\|R\|_{2}\right)\|x(t-h)\|_{2}^{2} \\
& -\left(\|\underline{A}\|_{2}\left\|K^{-2}\right\|_{2}-\chi\|V\|_{2}^{2}-\|V\|_{2}^{2}\left\|T^{-1}\right\|_{2}-\gamma_{2}-\beta_{2}\right) \\
& \times\|g(x(t))\|_{2}^{2} \\
& -\left(\beta_{2}\left(1-\mu_{2}\right)+\gamma_{2}\left(1-\mu_{2}\right)\right. \\
& \left.-\delta\left(1-\mu_{2}\right)\left\|V^{\sigma}\right\|_{2}^{2}-\left\|V^{\sigma}\right\|_{2}^{2}\left\|T^{-1}\right\|_{2}\right) \\
& \times\|g(x(t-\sigma(t)))\|_{2}^{2} \\
& -\left(\|\underline{B}\|_{2}-\left\|H_{2}\right\|_{2}-\frac{1}{\chi}-\frac{1}{\delta\left(1-\mu_{2}\right)}-\|B\|_{2}^{2}\left\|T^{-1}\right\|_{2}\right) \\
& \times\|y(t)\|_{2}^{2}-\left(\left\|H_{2}\right\|_{2}-3\|\Sigma\|_{2}^{2}\|T\|_{2}\right)\|y(t-d)\|_{2}^{2} \\
& -\left(\|\underline{B}\|_{2}\left\|L^{-2}\right\|_{2}-\alpha\|W\|_{2}^{2}-\|W\|_{2}^{2}\left\|R^{-1}\right\|_{2}-\gamma_{1}-\beta_{1}\right) \\
& \times\|f(y(t))\|_{2}^{2} \\
& -\left(\beta_{1}\left(1-\mu_{1}\right)+\gamma_{1}\left(1-\mu_{1}\right)-\beta\left(1-\mu_{1}\right)\left\|W^{\tau}\right\|_{2}^{2}\right. \\
& \left.-\left\|W^{\tau}\right\|_{2}^{2}\left\|R^{-1}\right\|_{2}\right) \\
& \times\|f(y(t-\tau(t)))\|_{2}^{2} \text {. }
\end{aligned}
$$

Let $\beta_{1}=\beta\left\|W^{\tau}\right\|_{2}^{2}, \gamma_{1}=\left(1 /\left(1-\mu_{1}\right)\right)\left\|W^{\tau}\right\|_{2}^{2}\left\|R^{-1}\right\|_{2}, \beta_{2}=$ $\delta\left\|V^{\sigma}\right\|_{2}^{2}$, and $\gamma_{2}=\left(1 /\left(1-\mu_{2}\right)\right)\left\|V^{\sigma}\right\|_{2}^{2}\left\|T^{-1}\right\|_{2}, \dot{V}(x(t), y(t))$ can be written in the form

$$
\begin{gathered}
\dot{V}(x(t), y(t)) \\
\leq- \\
\quad\left(\|\underline{A}\|_{2}-\left\|H_{1}\right\|_{2}-\frac{1}{\alpha}-\frac{1}{\beta\left(1-\mu_{1}\right)}-\|A\|_{2}^{2}\left\|R^{-1}\right\|_{2}\right) \\
-\left(\|x(t)\|_{2}^{2}-\left(\left\|H_{1}\right\|_{2}-3\|E\|_{2}^{2}\|R\|_{2}\right)\|x(t-h)\|_{2}^{2}\right. \\
\left.\quad-\frac{1}{\left(1-\mu_{2}\right)}\left\|V^{-2}\right\|_{2}-\chi\|V\|_{2}^{2}-\|V\|_{2}^{2}\left\|T^{-1}\right\|_{2}-\delta\left\|V_{2}^{\sigma}\right\|_{2}^{2}\right)\|g(x(t))\|_{2}^{2}
\end{gathered}
$$




$$
\begin{aligned}
& -\left(\|\underline{B}\|_{2}-\left\|H_{2}\right\|_{2}-\frac{1}{\chi}-\frac{1}{\delta\left(1-\mu_{2}\right)}-\|B\|_{2}^{2}\left\|T^{-1}\right\|_{2}\right) \\
& \times\|y(t)\|_{2}^{2}-\left(\left\|H_{2}\right\|_{2}-3\|\Sigma\|_{2}^{2}\|T\|_{2}\right)\|y(t-d)\|_{2}^{2} \\
& -\left(\|\underline{B}\|_{2}\left\|L^{-2}\right\|_{2}-\alpha\|W\|_{2}^{2}-\|W\|_{2}^{2}\left\|R^{-1}\right\|_{2}\right. \\
& \left.-\frac{1}{\left(1-\mu_{1}\right)}\left\|W^{\tau}\right\|_{2}^{2}\left\|R^{-1}\right\|_{2}-\beta\left\|W^{\tau}\right\|_{2}^{2}\right)\|f(y(t))\|_{2}^{2} .
\end{aligned}
$$

By Fact 1, $\|A\|_{2} \leq \sigma(A),\|B\|_{2} \leq \sigma(B),\|W\|_{2} \leq \sigma(W)$, $\left\|W^{\tau}\right\|_{2} \leq \sigma\left(W^{\tau}\right),\|V\|_{2} \leq \sigma(V)$, and $\left\|V^{\sigma}\right\|_{2} \leq \sigma\left(V^{\sigma}\right)$, one can have

$$
\begin{aligned}
\dot{V}(x(t), y(t)) & \\
\leq & -\left(\|\underline{A}\|_{2}-\left\|H_{1}\right\|_{2}-\frac{1}{\alpha}-\frac{1}{\beta\left(1-\mu_{1}\right)}-\sigma^{2}(A)\left\|R^{-1}\right\|_{2}\right) \\
& \times\|x(t)\|_{2}^{2}-\left(\left\|H_{1}\right\|_{2}-3 \sigma^{2}(E)\|R\|_{2}\right)\|x(t-h)\|_{2}^{2} \\
& -\left(\|\underline{A}\|_{2}\left\|K^{-2}\right\|_{2}-\chi \sigma^{2}(V)-\sigma^{2}(V)\left\|T^{-1}\right\|_{2}\right. \\
& \left.\quad-\frac{1}{\left(1-\mu_{2}\right)} \sigma^{2}\left(V^{\sigma}\right)\left\|T^{-1}\right\|_{2}-\delta \sigma^{2}\left(V^{\sigma}\right)\right) \\
& -\left(\|\underline{g}(x(t))\|_{2}^{2}-\left\|H_{2}\right\|_{2}-\frac{1}{\chi}-\frac{1}{\delta\left(1-\mu_{2}\right)}-\sigma^{2}(B)\left\|T^{-1}\right\|_{2}\right) \\
& \times\|y(t)\|_{2}^{2}-\left(\left\|H_{2}\right\|_{2}-3 \sigma^{2}(\Sigma)\|T\|_{2}\right)\|y(t-d)\|_{2}^{2} \\
& -\left({ }_{2}\left\|L^{-2}\right\|_{2}-\alpha \sigma^{2}(W)-\sigma^{2}(W)\left\|R^{-1}\right\|_{2}\right. \\
& \left.-\frac{1}{\left(1-\mu_{1}\right)} \sigma^{2}\left(W^{\tau}\right)\left\|R^{-1}\right\|_{2}-\beta \sigma^{2}\left(W^{\tau}\right)\right) \\
\times & \|f(y(t))\|_{2}^{2},
\end{aligned}
$$

or equivalently

$$
\begin{aligned}
\dot{V}(x(t), y(t)) \leq & -\theta_{1}\|x(t)\|_{2}^{2}-\theta_{2}\|x(t-h)\|_{2}^{2} \\
& -\theta_{3}\|g(x(t))\|_{2}^{2}-\theta_{4}\|y(t)\|_{2}^{2} \\
& -\theta_{5}\|y(t-d)\|_{2}^{2}-\theta_{6}\|f(y(t))\|_{2}^{2} .
\end{aligned}
$$

Clearly, $\theta_{i}>0$ and $i=1,2, \ldots, 6$, imply that $\dot{V}(x(t), y(t))<0$. On the other hand, $V(x(t), y(t)) \rightarrow$ $\infty$ as $x(t) \rightarrow \infty, y(t) \rightarrow \infty$, meaning that the Lyapunov functional used for the stability analysis is radially unbounded. Then, by the standard Lyapunov functional theory, it is concluded that system (8) or equivalently the equilibrium point of system (3) is globally asymptotically stable. This completes the proof of Theorem 6.

Remark 7. The stability results presented $[18,36,37]$ considered a pure delayed neural network mode and are expressed in the linear matrix inequality (LMI) forms. The LMI approach to the stability problem of neutral-type neural networks involves some difficulties with determining the constraint conditions on the network parameters as it requires testing positive definiteness of high dimensional matrices. However, Theorem 6 considers hybrid BAM neural networks and establishes various relationships between the network parameters only. Therefore, the results of this paper are applicable to a larger class of neural networks and can be easily verified when compared with the previously reported literature results.

Choosing $H_{1}, H_{2}, R$, and $T$ in the conditions of Theorem 6 as $H_{1}=h_{1} I, H_{2}=h_{2} I, R=r I$, and $T=t I$, we can express some special cases of Theorem 6 as follows.

Corollary 8. For given scalars $0 \leq \mu_{1} \leq 1$ and $0 \leq \mu_{2} \leq 1$, let the activation functions satisfy assumptions (H2) and (H3) and let the network parameters satisfy (4). Then, the origin of neural network model (8) is globally asymptotically stable, if there exist eight positive scalars $\alpha, \beta, \chi, \delta, h_{1}, h_{2}, r$, and $t$, such that

$$
\begin{aligned}
& \theta_{1}^{*}=\|\underline{A}\|_{2}-h_{1}-\frac{1}{\alpha}-\frac{1}{\beta\left(1-\mu_{1}\right)}-\sigma^{2}(A) \frac{1}{r}>0, \\
& \theta_{2}^{*}= h_{1}-3 \sigma^{2}(E) r>0, \\
& \theta_{3}^{*}=\|\underline{A}\|_{2}\left\|K^{-2}\right\|_{2}-\chi \sigma^{2}(V)-\sigma^{2}(V) \frac{1}{t} \\
& \quad-\frac{1}{\left(1-\mu_{2}\right)} \sigma^{2}\left(V^{\sigma}\right) \frac{1}{t}-\delta \sigma^{2}\left(V^{\sigma}\right)>0, \\
& \theta_{4}^{*}=\|\underline{B}\|_{2}-h_{2}-\frac{1}{\chi}-\frac{1}{\delta\left(1-\mu_{2}\right)}-\sigma^{2}(B) \frac{1}{t}>0, \\
& \theta_{5}^{*}=h_{2}-3 \sigma^{2}(\Sigma) t>0, \\
& \theta_{6}^{*}=\|\underline{B}\|_{2}\left\|L^{-2}\right\|_{2}-\alpha \sigma^{2}(W)-\sigma^{2}(W) \frac{1}{r}
\end{aligned}
$$$$
-\frac{1}{\left(1-\mu_{1}\right)} \sigma^{2}\left(W^{\tau}\right) \frac{1}{r}-\beta \sigma^{2}\left(W^{\tau}\right)>0,
$$

and the other parameters are defined in Theorem 6.

By setting $\mu_{1}=\mu_{2}=0$, the stability criterion for hybrid BAM neural network with constant time delays is established from Theorem 6 .

Corollary 9. Let the activation functions satisfy assumptions (H2) and (H3) and let the network parameters satisfy (4). Then, the origin of neural network model (8) is globally 
asymptotically stable, if there exists eight positive scalars $\alpha, \beta$, $\chi, \delta, h_{1}, h_{2}, r$, and $t$, such that

$$
\begin{aligned}
& \theta_{1}^{* *}=\|\underline{A}\|_{2}-h_{1}-\frac{1}{\alpha}-\frac{1}{\beta}-\sigma^{2}(A) \frac{1}{r}>0, \theta_{2}^{* *}=h_{1}-3 \sigma^{2}(E) r>0, \\
& \theta_{3}^{* *}=\|\underline{A}\|_{2}\left\|K^{-2}\right\|_{2}-\chi \sigma^{2}(V)-\sigma^{2}(V) \frac{1}{t} \\
& \quad-\sigma^{2}\left(V^{\sigma}\right) \frac{1}{t}-\delta \sigma^{2}\left(V^{\sigma}\right)>0, \\
& \theta_{4}^{* *}=\|\underline{B}\|_{2}-h_{2}-\frac{1}{\chi}-\frac{1}{\delta}-\sigma^{2}(B) \frac{1}{t}>0, \\
& \theta_{5}^{* *}=h_{2}-3 \sigma^{2}(\Sigma) t>0, \\
& \theta_{6}^{* *}=\|\underline{B}\|_{2}\left\|L^{-2}\right\|_{2}-\alpha \sigma^{2}(W)-\sigma^{2}(W) \frac{1}{r} \\
& \quad-\frac{1}{\left(1-\mu_{1}\right)} \sigma^{2}\left(W^{\tau}\right) \frac{1}{r}-\beta \sigma^{2}\left(W^{\tau}\right)>0,
\end{aligned}
$$

and the other parameters are defined in Theorem 6.

Assume that there are no neutral terms and the system of BAM neural networks is described as

$$
\begin{array}{r}
\dot{x}(t)=-A x(t)+W f(y(t))+W^{\tau} f(y(t-\tau(t))), \\
i=1,2, \ldots, n, \\
\dot{y}(t)=-B y(t)+V g(x(t))+V^{\sigma} g(x(t-\sigma(t))), \\
j=1,2, \ldots, m .
\end{array}
$$
tional:

Define the following positive definite Lyapunov func-

$$
\begin{aligned}
V(x(t), y(t))= & x^{T}(t) x(t) y(t)^{T} y(t)+\sum_{i=1}^{n} h_{1 i} \int_{t-h}^{t} x_{i}^{2}(s) d s \\
& +\sum_{j=1}^{m} h_{2 j} \int_{t-d}^{t} y_{j}^{2}(s) d s \\
& +\beta_{1} \sum_{j=1}^{m} \int_{t-\tau(t)}^{t} f_{j}\left(y_{j}(\xi)\right) d \xi \\
& +\beta_{2} \sum_{i=1}^{n} \int_{t-\sigma(t)}^{t} g_{i}\left(x_{i}(\xi)\right) d \xi .
\end{aligned}
$$

Following the similar line of the proof of Theorem 6 , Corollary 10 is derived as follows.

Corollary 10. For given scalars $0 \leq \mu_{1} \leq 1$, and $0 \leq \mu_{2} \leq 1$, let the activation functions satisfy assumptions (H2) and (H3) and let the network parameters satisfy (4). Then, the origin of neural network model (8) is globally asymptotically stable, if there exist four positive scalars $\alpha, \beta, \chi$, and $\delta$, such that

$$
\begin{aligned}
& \eta_{1}=\|\underline{A}\|_{2}-\frac{1}{\alpha}-\frac{1}{\beta\left(1-\mu_{1}\right)}>0, \\
& \eta_{2}=\|\underline{A}\|_{2}\left\|K^{-2}\right\|_{2}-\chi \sigma^{2}(V)-\delta \sigma^{2}\left(V^{\sigma}\right)>0, \\
& \eta_{3}=\|\underline{B}\|_{2}-\frac{1}{\chi}-\frac{1}{\delta\left(1-\mu_{2}\right)}>0, \\
& \eta_{4}=\|\underline{B}\|_{2}\left\|L^{-2}\right\|_{2}-\alpha \sigma^{2}(W)-\beta \sigma^{2}\left(W^{\tau}\right)>0,
\end{aligned}
$$

and the other parameters are defined in Theorem 6.

\section{Comparative Numerical Examples}

We will now give the following examples to demonstrate the applicability and advantages of our results.

Example 11. Assume that the network parameters of neural system (8) are given as follows:

$$
\begin{gathered}
\underline{W}=\underline{W}^{\tau}=\underline{V}=\underline{V}^{\sigma}=\left[\begin{array}{ccc}
3 \lambda & 3 \lambda & 4 \lambda \\
6 \lambda & 2 \lambda & 7 \lambda \\
-7 \lambda & 7 \lambda & -6 \lambda
\end{array}\right], \\
\bar{W}=\bar{W}^{\tau}=\bar{V}=\bar{V}^{\sigma}=\left[\begin{array}{ccc}
7 \lambda & 5 \lambda & 4 \lambda \\
8 \lambda & 4 \lambda & 9 \lambda \\
-3 \lambda & 7 \lambda & -2 \lambda
\end{array}\right], \\
\underline{A}=A=\bar{A}=\underline{B}=B=\bar{B}=\left[\begin{array}{lll}
3 & 0 & 0 \\
0 & 3 & 0 \\
0 & 0 & 3
\end{array}\right], \\
K=L=\left[\begin{array}{lll}
1 & 0 & 0 \\
0 & 1 & 0 \\
0 & 0 & 1
\end{array}\right]
\end{gathered}
$$

where $\lambda=0$ is real number. We can conclude that the matrices $W^{*}, W_{*}, W^{\tau *}, W_{*}^{\tau}, V^{*}, V_{*}, V^{\sigma *}$, and $V_{*}^{\sigma}$ are in the forms

$$
\begin{gathered}
\|A\|_{2}=\|B\|_{2}=3, \\
W^{*}=W^{\tau *}=V^{*}=V^{\sigma *}=\left[\begin{array}{ccc}
5 \lambda & 4 \lambda & 4 \lambda \\
7 \lambda & 3 \lambda & 8 \lambda \\
-5 \lambda & 7 \lambda & -4 \lambda
\end{array}\right], \\
W_{*}=W_{*}^{\tau}=V_{*}=V_{*}^{\sigma}=\left[\begin{array}{ccc}
2 \lambda & \lambda & 0 \\
\lambda & \lambda & \lambda \\
2 \lambda & 0 & 0
\end{array}\right], \\
\tau=2, \quad \sigma=3, \quad h=1, \\
d=2, \quad \mu_{1}=0, \quad \mu_{2}=0 .
\end{gathered}
$$


Then we obtain

$$
\begin{aligned}
\sigma_{1}\left(V^{\sigma}\right) & =\sigma_{1}\left(W^{\tau}\right)=\sigma_{1}(V)=\sigma_{1}(W) \\
& =\sqrt{\left\|\left|W^{* T} W^{*}\right|+2\left|W^{* T}\right| W_{*}+W_{*}^{T} W_{*}\right\|_{2}} \\
& =\sqrt{272.7882} \lambda^{2}=16.5163 \lambda, \\
\sigma_{2}\left(V^{\sigma}\right) & =\sigma_{2}\left(W^{\tau}\right)=\sigma_{2}(V)=\sigma_{2}(W) \\
& =\left\|W^{*}\right\|_{2}+\left\|W_{*}\right\|_{2}=17.5942 \lambda . \\
\sigma_{3}\left(V^{\sigma}\right) & =\sigma_{3}\left(W^{\tau}\right)=\sigma_{3}(V)=\sigma_{3}(W) \\
& =\sqrt{\left\|W^{*}\right\|_{2}^{2}+\left\|W_{*}\right\|_{2}^{2}+2\left\|W_{*}^{T}\left|W^{*}\right|\right\|_{2}} \\
& =\sqrt{308.2903} \lambda^{2}=17.5582 \lambda . \\
\sigma_{4}\left(V^{\sigma}\right) & =\sigma_{4}\left(W^{\tau}\right)=\sigma_{4}(V) \\
& =\sigma_{4}(W)=\|\widehat{W}\|_{2} \\
& =19.2861 \lambda .
\end{aligned}
$$

Since $\min \left\{\sigma_{1}(W), \sigma_{2}(W), \sigma_{3}(W), \sigma_{4}(W)\right\}=16.5163 \lambda$, we obtain $\sigma(V)=\sigma(W)=\sigma\left(V^{\sigma}\right)=\sigma\left(W^{\tau}\right)=16.5163 \lambda$.

For the sufficiently small values of $\|E\|_{2},\|\Sigma\|_{2}, h_{1}$, and $h_{2}$ and sufficiently large value of $r, t$, and $\alpha=\beta, \chi=\delta$, the conditions of Corollary 9 can be approximately stated as follows: $\theta_{6}^{* *}=3-2 \alpha \times 272.7882 \lambda^{2}>0, \theta_{6}^{* *} \cong 3-2 \alpha \times$ $272.7882 \lambda^{2}>0$, and

$$
\begin{aligned}
& \theta_{1}^{* *} \cong 3-\frac{2}{\alpha}>0, \\
& \theta_{2}^{* *} \cong h_{1}-3 \sigma^{2}(E) r>0, \\
& \theta_{3}^{* *} \cong 3-2 \chi \times 272.7882 \lambda^{2}>0, \\
& \theta_{4}^{* *} \cong 3-\frac{2}{\chi}>0, \\
& \theta_{5}^{* *} \cong h_{2}-3 \sigma^{2}(F) t>0, \\
& \theta_{6}^{* *} \cong 3-2 \alpha \times 272.7882 \lambda^{2}>0 .
\end{aligned}
$$

The four required conditions for stability are $\alpha>2 / 3, \chi>$ $2 / 3$ and $\lambda^{2}<3 /(2 \chi \times 272.7882), \lambda^{2}<3 /(2 \alpha \times 272.7882)$, implying that $\lambda<0.0908$. Hence, if $\lambda<0.0908$ holds, then the conditions of Corollary 9 are satisfied which indicates that the BAM neural network is global asymptotic robust stable.

In what follows, we consider a special model in this example and give simulation results for the sake of verification of our proposed results. We choose $\lambda=0.06$ that satisfies the condition $\lambda<0.0908$. For this example, the Matlab simulation results are presented in Figure 1.
Example 12. Assume that the network parameters of neural system (8) are given as follows:

$$
\begin{aligned}
& \underline{A}=A=\bar{A}=\underline{B}=B=\bar{B}=I, \\
& \underline{W}=\left[\begin{array}{cc}
0.2 & 0.1 \\
-0.1 & 0.2
\end{array}\right], \quad \bar{W}=\left[\begin{array}{cc}
0.4 & 0.1 \\
0.1 & 0.4
\end{array}\right], \\
& \underline{W}^{\tau}=\left[\begin{array}{ll}
0.1 & 0.2 \\
0.2 & 0.2
\end{array}\right], \quad \bar{W}^{\tau}=\left[\begin{array}{ll}
0.3 & 0.4 \\
0.3 & 0.2
\end{array}\right], \\
& \underline{V}=\left[\begin{array}{cc}
0.1 & 0.1 \\
-0.3 & 0.1
\end{array}\right], \quad \bar{V}=\left[\begin{array}{cc}
0.3 & 0.3 \\
0.1 & 0.3
\end{array}\right] \text {, } \\
& \underline{V}^{\sigma}=\left[\begin{array}{ll}
0.1 \lambda & 0.1 \lambda \\
0.1 \lambda & 0.2 \lambda
\end{array}\right], \quad \underline{V}^{\sigma}=\left[\begin{array}{ll}
0.3 \lambda & 0.3 \lambda \\
0.3 \lambda & 0.4 \lambda
\end{array}\right], \\
& \underline{E}=\left[\begin{array}{ll}
0.01 & 0.01 \\
0.01 & 0.01
\end{array}\right], \quad \bar{E}=\left[\begin{array}{ll}
0.05 & 0.05 \\
0.05 & 0.05
\end{array}\right], \\
& \underline{\Sigma}=\left[\begin{array}{ll}
0.01 & 0.01 \\
0.01 & 0.01
\end{array}\right], \quad \bar{\Sigma}=\left[\begin{array}{ll}
0.05 & 0.05 \\
0.05 & 0.05
\end{array}\right], \\
& K=\Sigma=0.5 I, \quad \tau(t)=0.5 \sin t+0.1, \\
& \sigma(t)=0.5 \sin t+0.2, \quad \mu_{1}=\mu_{2}=0.5,
\end{aligned}
$$

where $\lambda>0$ is real number. We can obtain

$$
\begin{array}{cc}
\|A\|_{2}=\|B\|_{2}=1, & \sigma(A)=\sigma(B)=1, \\
W^{*}=\left[\begin{array}{cc}
0.3 & 0.1 \\
0 & 0.3
\end{array}\right], & W_{*}=\left[\begin{array}{cc}
0.1 & 0 \\
0.1 & 0.1
\end{array}\right], \\
W^{\tau *}=\left[\begin{array}{cc}
0.2 & 0.3 \\
0.25 & 0.2
\end{array}\right], & W_{*}^{\sigma}=\left[\begin{array}{cc}
0.1 & 0.1 \\
0.05 & 0.2
\end{array}\right], \\
V^{*}=\left[\begin{array}{cc}
0.3 & 0.1 \\
0 & 0.3
\end{array}\right], & V_{*}=\left[\begin{array}{cc}
0.1 & 0 \\
0.1 & 0.1
\end{array}\right], \\
V^{\sigma *}=\left[\begin{array}{cc}
0.4 \lambda & 0.3 \lambda \\
0.25 \lambda & 0.2 \lambda
\end{array}\right], & V_{*}^{\sigma}=\left[\begin{array}{cc}
0.3 \lambda & 0.1 \lambda \\
0.05 \lambda & 0.2 \lambda
\end{array}\right], \\
E^{*}=\left[\begin{array}{cc}
0.03 & 0.03 \\
0.03 & 0.03
\end{array}\right], & E_{*}=\left[\begin{array}{ll}
0.02 & 0.02 \\
0.02 & 0.02
\end{array}\right], \\
\Sigma^{*}=\left[\begin{array}{ll}
0.03 & 0.03 \\
0.03 & 0.03
\end{array}\right], & \Sigma_{*}=\left[\begin{array}{ll}
0.02 & 0.02 \\
0.02 & 0.02
\end{array}\right] .
\end{array}
$$

By Lemmas $1-4$, we can calculate

$$
\begin{aligned}
& \sigma_{1}(W)=\sqrt{\left\|\left|W^{* T} W^{*}\right|+2\left|W^{* T}\right| W_{*}+W_{*}^{T} W_{*}\right\|_{2}} \\
& =\sqrt{0.2546}, \quad \sigma_{2}(W)=\left\|W^{*}\right\|_{2}+\left\|W_{*}\right\|_{2}=0.5159 \text {, } \\
& \sigma_{3}(W)=\sqrt{\left\|W^{*}\right\|_{2}^{2}+\left\|W_{*}\right\|_{2}^{2}+2\left\|W_{*}^{T}\left|W^{*}\right|\right\|_{2}}=0.5 \text {, } \\
& \sigma_{4}(W)=\|\widehat{W}\|_{2}=0.2656 .
\end{aligned}
$$




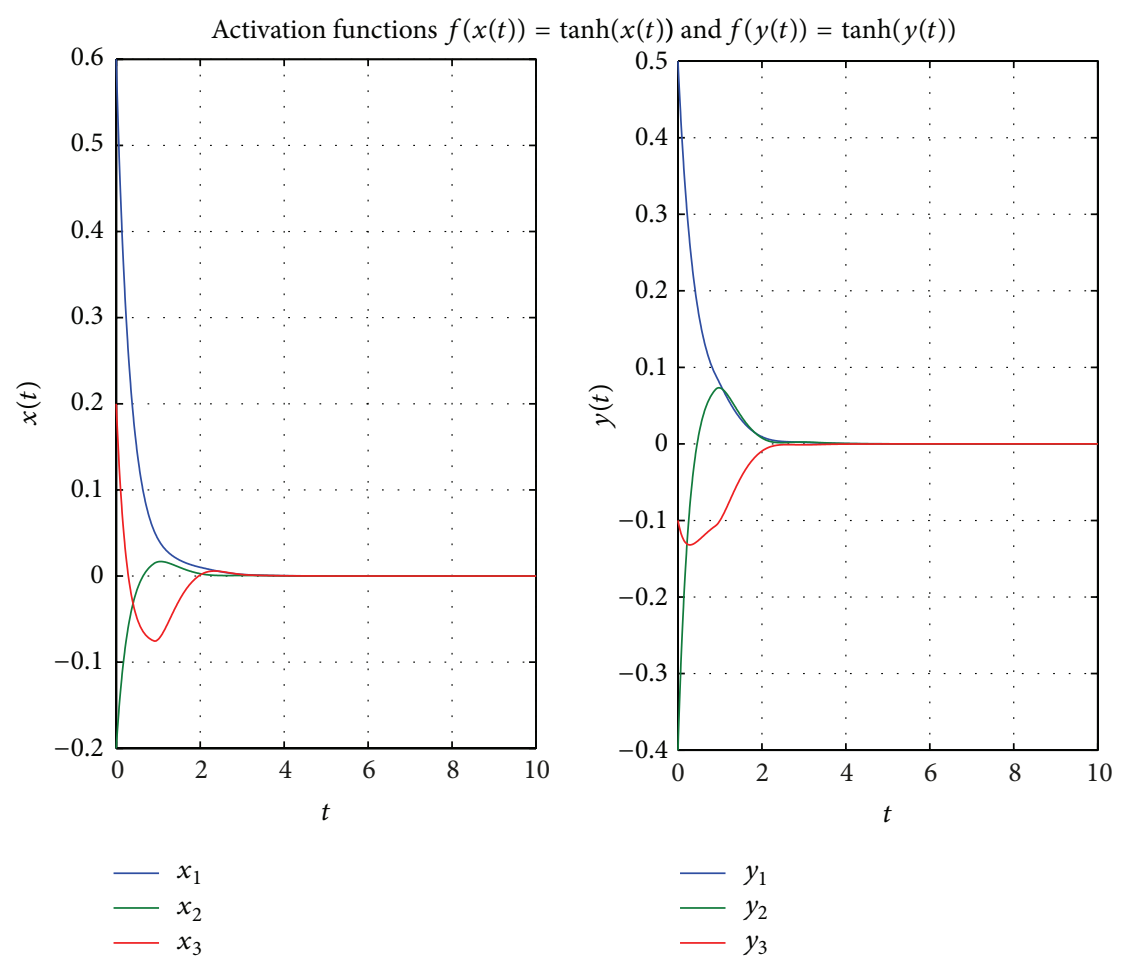

FIGURE 1: Trajectories of $x(t)$ and $y(t)$ of system $(8)$ for the initial states $x(0)=\left[\begin{array}{lll}0.6 & -0.2 & 0.2\end{array}\right]$ and $y(0)=\left[\begin{array}{lll}0.5 & -0.4 & -0.1\end{array}\right]$.

Since $\sigma(W)=\min \left\{\sigma_{1}(W), \sigma_{2}(W), \sigma_{3}(W), \sigma_{4}(W)\right\}$, we obtain $\sigma(W)=0.5$, Similarly, we obtain

$$
\begin{array}{cc}
\sigma_{1}\left(W^{\tau}\right)=\sqrt{0.5018}, & \sigma_{2}\left(W^{\tau}\right)=0.7188, \\
\sigma_{3}\left(W^{\tau}\right)=\sqrt{0.4967}, & \sigma_{4}\left(W^{\tau}\right)=0.6085, \\
\sigma_{1}(V)=\sqrt{0.3225}, & \sigma_{2}(V)=0.5618, \\
\sigma_{3}(V)=\sqrt{0.3440}, & \sigma_{4}(V)=0.6, \\
\sigma_{1}\left(V^{\sigma}\right)=\sqrt{0.4281} \lambda, & \sigma_{2}\left(V^{\sigma}\right)=0.6562 \lambda, \\
\sigma_{3}\left(V^{\sigma}\right)=\sqrt{0.4292} \lambda, & \sigma_{4}\left(V^{\sigma}\right)=0.6541 \lambda, \\
\sigma_{1}(E)=\sigma_{1}(\Sigma)=\sigma_{2}(E)=\sigma_{2}(\Sigma) \\
=\sigma_{3}(E)=\sigma_{3}(\Sigma)=\sigma_{4}(E)=\sigma_{4}(\Sigma)=0.1 .
\end{array}
$$

Thus we have $\sigma\left(W^{\tau}\right)=0.6085, \sigma(V)=0.5618, \sigma\left(V^{\sigma}\right)=$ $0.6541 \lambda$, and $\sigma(E)=\sigma(\Sigma)=0.1$.

Let $\alpha=\beta=6, \chi=\delta=6$, and $r=t=6$; the conditions of Corollary 8 can be stated as follows:

$$
\begin{aligned}
& \theta_{1}^{*}=1-h_{1}-\frac{1}{6}-\frac{1}{6(1-0.5)}-\frac{1}{6}>0, \\
& \theta_{2}^{*}=h_{1}-3 \times 0.01 \times 6>0,
\end{aligned}
$$

$$
\begin{aligned}
\theta_{3}^{*}= & 4-6 \times 0.3156-\frac{0.3156}{6} \\
& -\frac{1}{(1-0.5)} 0.6541^{2} \lambda^{2} \times \frac{1}{6}-6 \times 0.6541^{2} \lambda^{2}>0, \\
\theta_{4}^{*}= & 1-h_{2}-\frac{1}{4}-\frac{1}{4(1-0.5)}-\frac{1}{6}>0 \\
\theta_{5}^{*}= & h_{2}-3 \times 0.01 \times 6>0, \\
\theta_{6}^{*}= & 4-6 \times 0.25-0.25 \times \frac{1}{6}-\frac{1}{(1-0.5)} \\
& \times 0.3703 \times \frac{1}{6}-6 \times 0.3703=0.1131>0
\end{aligned}
$$

in which $\lambda<0.8706$ implies that the conditions of Corollary 8 are satisfied which indicates that the network is global asymptotic robust stable.

For the neural network parameters given in Example 12, we choose $\lambda=0.6$ that satisfies the condition $\lambda<0.8706$. For this example, the Matlab simulation results are presented in Figure 2.

\section{Conclusions}

In this paper, we have obtained new sufficient conditions for the global asymptotic robust stability of the equilibrium point for the class of neutral-type hybrid bidirectional associative memory neural networks with time-varying delays 


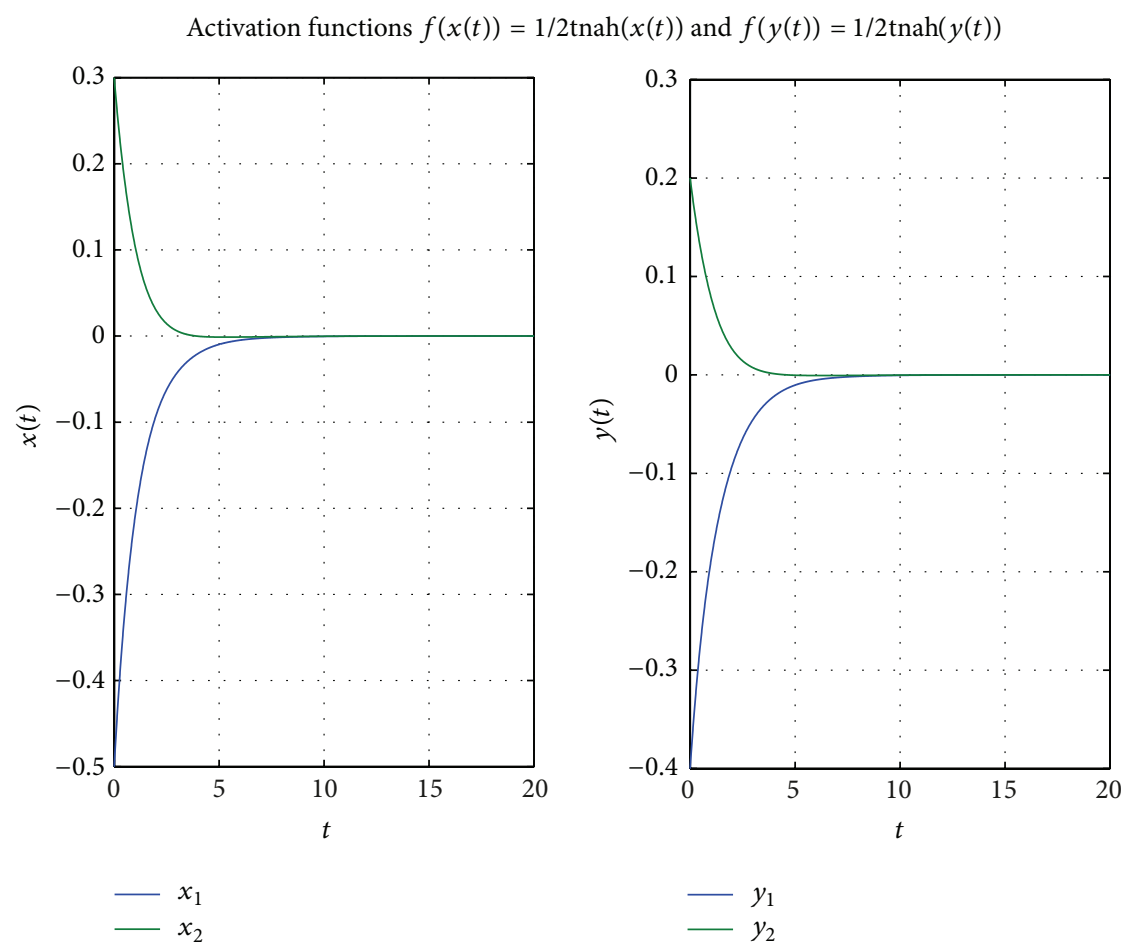

FIGURE 2: Trajectories of $x(t)$ and $y(t)$ of system (8) for the initial states $x(0)=\left[\begin{array}{ll}-0.5 & 0.3\end{array}\right]$ and $y(0)=\left[\begin{array}{ll}-0.4 & 0.2\end{array}\right]$.

and parameters uncertainties. Some new delay-derivativedependent stability criteria are derived to ascertain the global asymptotic stability of the BAM neural networks. To obtain less conservative stability criterion, some new upper bound norms for the interconnection matrices of the neural networks are used. The obtained results can be easily verified as they can be expressed in terms of the network parameters only. Two illustrative examples are given to show the effectiveness of the proposed results.

\section{Conflict of Interests}

The authors declare that there is no conflict of interests regarding the publication of this paper.

\section{Acknowledgments}

This work is supported by National Natural Science Foundation of China (no. 61103211) and Postdoctoral Science Foundation of Chongqing (no. XM201310).

\section{References}

[1] L. O. Chua, CNN: A Paradigm for Complexity, World Scientific Publishing, River Edge, NJ, USA, 1998.

[2] Y. Fang and T. G. Kincaid, "Stability analysis of dynamical neural networks," IEEE Transactions on Neural Networks, vol. 7, no. 4, pp. 996-1006.

[3] A. N. Michel and D. Liu, Qualitative Analysis and Synthesis of Recurrent Neural Networks, CRC Press, New York, NY, USA, 2001.
[4] B. Kosko, "Adaptive bidirectional associative memories," Applied Optics, vol. 26, no. 23, pp. 4947-4960, 1987.

[5] B. Kosko, "Bidirectional associative memories," IEEE Transactions on Systems, Man, and Cybernetics, vol. 18, no. 1, pp. 49-60, 1988.

[6] M. S. Mahmoud and Y. Xia, "LMI-based exponential stability criterion for bidirectional associative memory neural networks," Neurocomputing, vol. 74, no. 1-3, pp. 284-290, 2010.

[7] H. Wang, Q. Song, and C. Duan, "LMI criteria on exponential stability of BAM neural networks with both time-varying delays and general activation functions," Mathematics and Computers in Simulation, vol. 81, no. 4, pp. 837-850, 2010.

[8] Z. Zhang and D. Zhou, "Existence and global exponential stability of a periodic solution for a discrete-time interval general BAM neural networks," Journal of the Franklin Institute, vol. 347, no. 5, pp. 763-780, 2010.

[9] P. Balasubramaniam and M. S. Ali, "Stability analysis of TakagiSugeno fuzzy Cohen-Grossberg BAM neural networks with discrete and distributed time-varying delays," Mathematical and Computer Modelling, vol. 53, no. 1-2, pp. 151-160, 2011.

[10] Z. Luo, J. Li, and J. Shen, "Stability of bidirectional associative memory networks with impulses," Applied Mathematics and Computation, vol. 218, no. 5, pp. 1658-1667, 2011.

[11] R. Raja and S. M. Anthoni, "Global exponential stability of BAM neural networks with time-varying delays: the discretetime case," Communications in Nonlinear Science and Numerical Simulation, vol. 16, no. 2, pp. 613-622, 2011.

[12] Z. Zhang and K. Liu, "Existence and global exponential stability of a periodic solution to interval general bidirectional associative memory (BAM) neural networks with multiple delays on time scales," Neural Networks, vol. 24, no. 5, pp. 427-439, 2011.

[13] Q. Zhu, X. Li, and X. Yang, "Exponential stability for stochastic reaction-diffusion BAM neural networks with time-varying 
and distributed delays," Applied Mathematics and Computation, vol. 217, no. 13, pp. 6078-6091, 2011.

[14] Y. Huang, H. Zhang, and Z. Wang, "Multistability and multiperiodicity of delayed bidirectional associative memory neural networks with discontinuous activation functions," Applied Mathematics and Computation, vol. 219, no. 3, pp. 899-910, 2012.

[15] B. Liu, "Global exponential stability for BAM neural networks with time-varying delays in the leakage terms," Nonlinear Analysis: Real World Applications, vol. 14, no. 1, pp. 559-566, 2013.

[16] Z. Zhao, F. Liu, X. Xie, X. Liu, and Z. Tang, "Asymptotic stability of bidirectional associative memory neural networks with timevarying delays via delta operator approach," Neurocomputing, vol. 117, pp. 40-46.

[17] A. Zhang, J. Qiu, and J. She, "Existence and global exponential stability of periodic solution for high-order discrete-time BAM neural networks," Neural Networks, vol. 50, pp. 98-109.

[18] Z. Zhang, K. Liu, and Y. Yang, "New LMI-based condition on global asymptotic stability concerning BAM neural networks of neutral type," Neurocomputing, vol. 81, pp. 24-32, 2012.

[19] Q. Song and J. Cao, "Dynamics of bidirectional associative memory networks with distributed delays and reactiondiffusion terms," Nonlinear Analysis: Real World Applications, vol. 8, no. 1, pp. 345-361, 2007.

[20] X. Liao and K.-W. Wong, "Robust stability of interval bidirectional associative memory neural network with time delays," IEEE Transactions on Systems, Man, and Cybernetics B: Cybernetics, vol. 34, no. 2, pp. 1142-1154, 2004.

[21] X. Lou and B. Cui, "On the global robust asymptotic stability of BAM neural networks with time-varying delays," Neurocomputing, vol. 70, no. 1-3, pp. 273-279, 2006.

[22] J. H. Park, "Robust stability of bidirectional associative memory neural networks with time delays," Physics Letters A: General, Atomic and Solid State Physics, vol. 349, no. 6, pp. 494-499, 2006.

[23] S. Senan and S. Arik, "Global robust stability of bidirectional associative memory neural networks with multiple time delays," IEEE Transactions on Systems, Man, and Cybernetics B: Cybernetics, vol. 37, no. 5, pp. 1375-1381, 2007.

[24] M. Gao and B. Cui, "Global robust exponential stability of discrete-time interval BAM neural networks with time-varying delays," Applied Mathematical Modelling, vol. 33, no. 3, pp. 12701284, 2009.

[25] S. Senan and S. Arik, "New results for global robust stability of bidirectional associative memory neural networks with multiple time delays," Chaos, Solitons \& Fractals, vol. 41, no. 4, pp. 2106-2114, 2009.

[26] L. Sheng and H. Yang, "Novel global robust exponential stability criterion for uncertain BAM neural networks with time-varying delays," Chaos, Solitons \& Fractals, vol. 40, no. 5, pp. 2102-2113, 2009.

[27] M. S. Ali and P. Balasubramaniam, "Robust stability of uncertain fuzzy Cohen-Grossberg BAM neural networks with timevarying delays," Expert Systems with Applications, vol. 36, no. 7, pp. 10583-10588, 2009.

[28] Y. Yuan and X. Li, "New results for global robust asymptotic stability of BAM neural networks with time-varying delays," Neurocomputing, vol. 74, no. 1-3, pp. 337-342, 2010.

[29] Q. Zhou and L. Wan, "Global robust asymptotic stability analysis of BAM neural networks with time delay and impulse: an LMI approach," Applied Mathematics and Computation, vol. 216, no. 5, pp. 1538-1545, 2010.
[30] S. Senan, S. Arik, and D. Liu, "New robust stability results for bidirectional associative memory neural networks with multiple time delays," Applied Mathematics and Computation, vol. 218, no. 23, pp. 11472-11482, 2012.

[31] X. Li and J. Jia, "Global robust stability analysis for BAM neural networks with time-varying delays," Neurocomputing, vol. 120, pp. 499-503.

[32] J. Cao, D. W. C. Ho, and X. Huang, "LMI-based criteria for global robust stability of bidirectional associative memory networks with time delay," Nonlinear Analysis: Theory, Methods \& Applications, vol. 66, no. 7, pp. 1558-1572, 2007.

[33] J. Qiu and J. Cao, "Delay-dependent robust stability of neutraltype neural networks with time delays," Journal of Mathematical Control Science and Applications, vol. 1, no. 1, pp. 179-188.

[34] J.-E. Feng, S. Xu, and Y. Zou, "Delay-dependent stability of neutral type neural networks with distributed delays," Neurocomputing, vol. 72, no. 10-12, pp. 2576-2580, 2009.

[35] Z. Zhang, W. Liu, and D. Zhou, "Global asymptotic stability to a generalized Cohen-Grossberg BAM neural networks of neutral type delays," Neural Networks, vol. 25, no. 1, pp. 94-105, 2012.

[36] J. H. Park, C. H. Park, O. M. Kwon, and S. M. Lee, "A new stability criterion for bidirectional associative memory neural networks of neutral-type," Applied Mathematics and Computation, vol. 199, no. 2, pp. 716-722, 2008.

[37] J. Liu and G. Zong, "New delay-dependent asymptotic stability conditions concerning BAM neural networks of neutral type," Neurocomputing, vol. 72, no. 10-12, pp. 2549-2555, 2009.

[38] N. Ozcan and S. Arik, "A new sufficient condition for global robust stability of bidirectional associative memory neural networks with multiple time delays," Nonlinear Analysis: Real World Applications, vol. 10, no. 5, pp. 3312-3320, 2009.

[39] O. Faydasicok and S. Arik, "A new upper bound for the norm of interval matrices with application to robust stability analysis of delayed neural networks," Neural Networks, vol. 44, pp. 67-71.

[40] J. Cao, D.-S. Huang, and Y. Qu, "Global robust stability of delayed recurrent neural networks," Chaos, Solitons \& Fractals, vol. 23, no. 1, pp. 221-229, 2005.

[41] T. Ensari and S. Arik, "New results for robust stability of dynamical neural networks with discrete time delays," Expert Systems with Applications, vol. 37, no. 8, pp. 5925-5930, 2010.

[42] V. Singh, "Global robust stability of delayed neural networks: estimating upper limit of norm of delayed connection weight matrix," Chaos, Solitons \& Fractals, vol. 32, no. 1, pp. 259-263, 2007. 


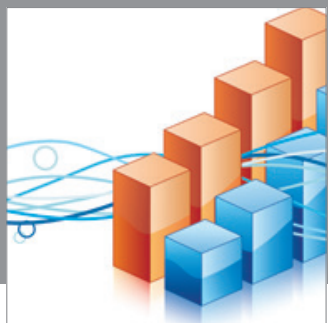

Advances in

Operations Research

mansans

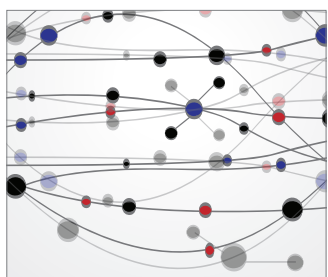

The Scientific World Journal
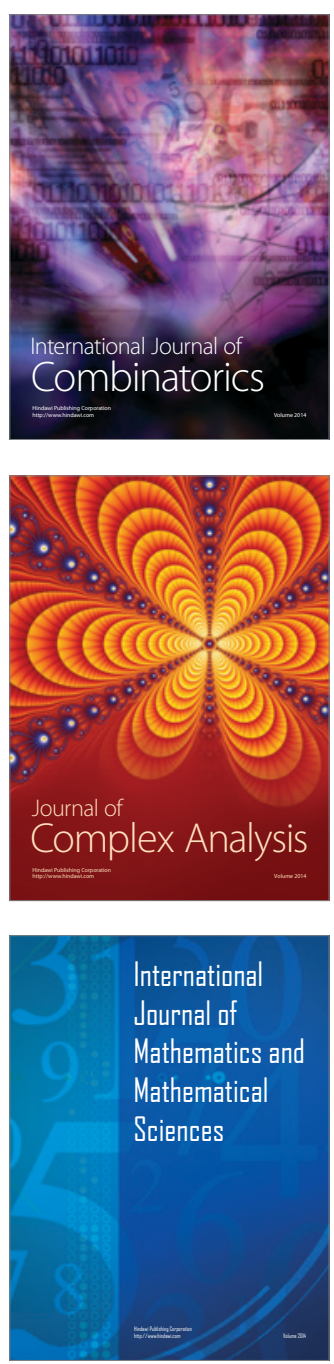
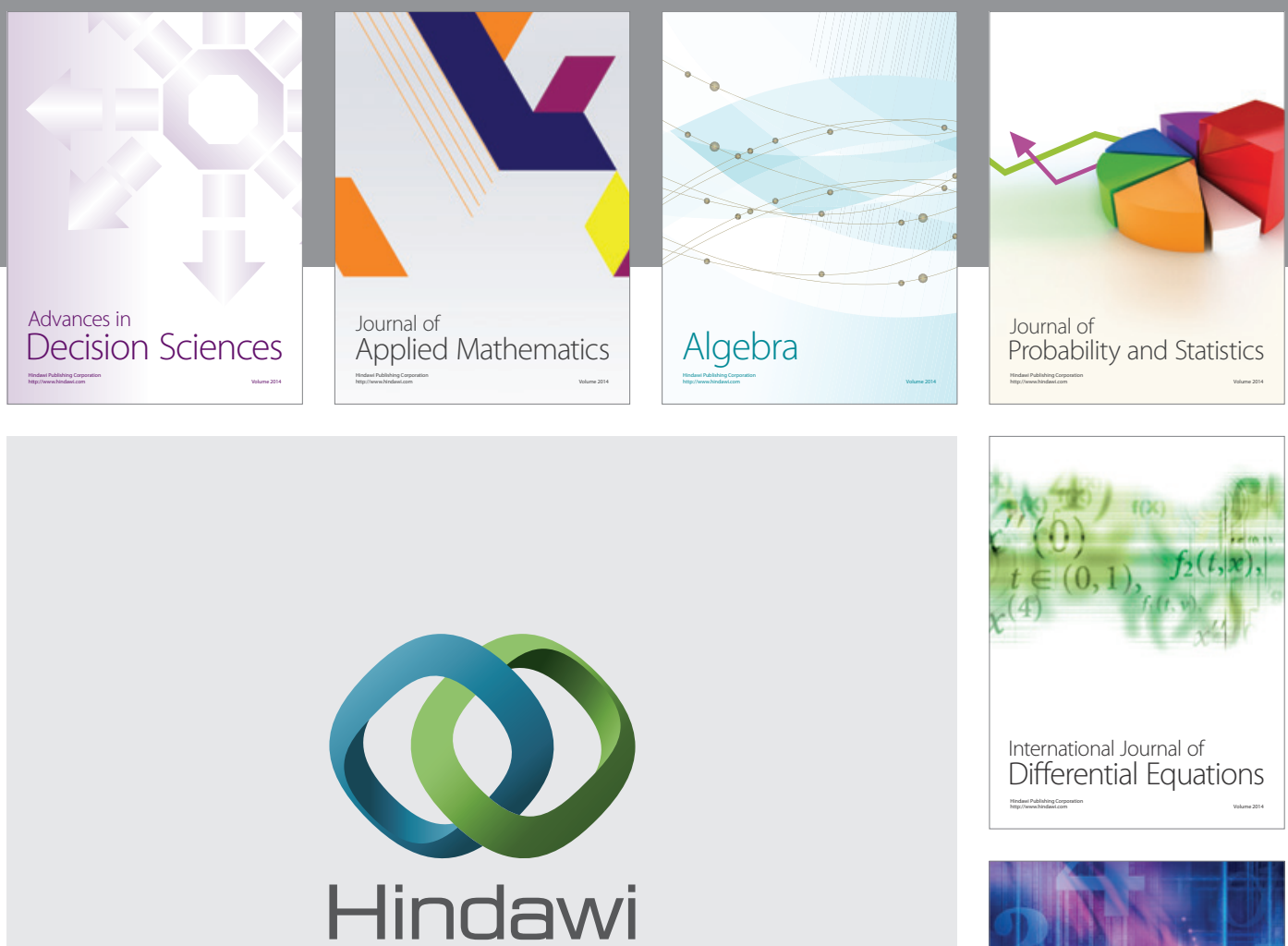

Submit your manuscripts at http://www.hindawi.com
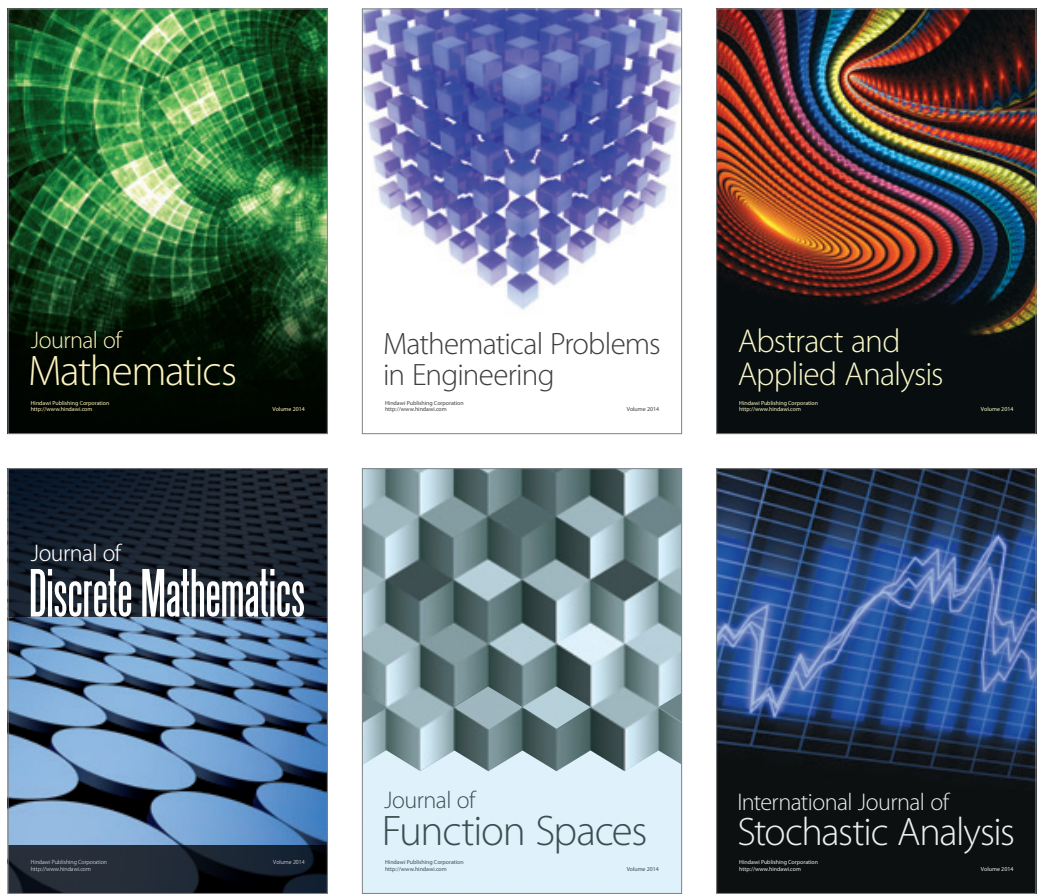

Journal of

Function Spaces

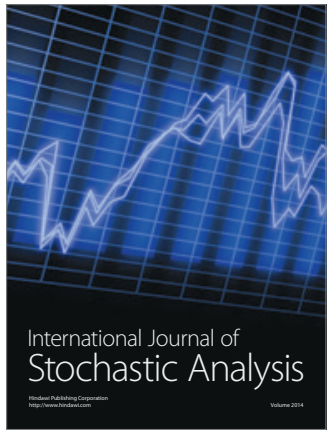

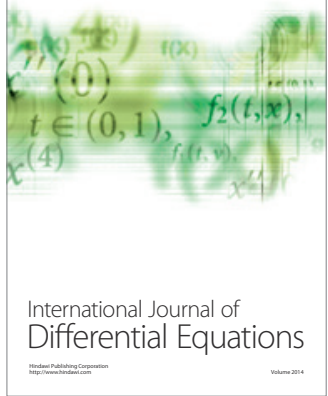
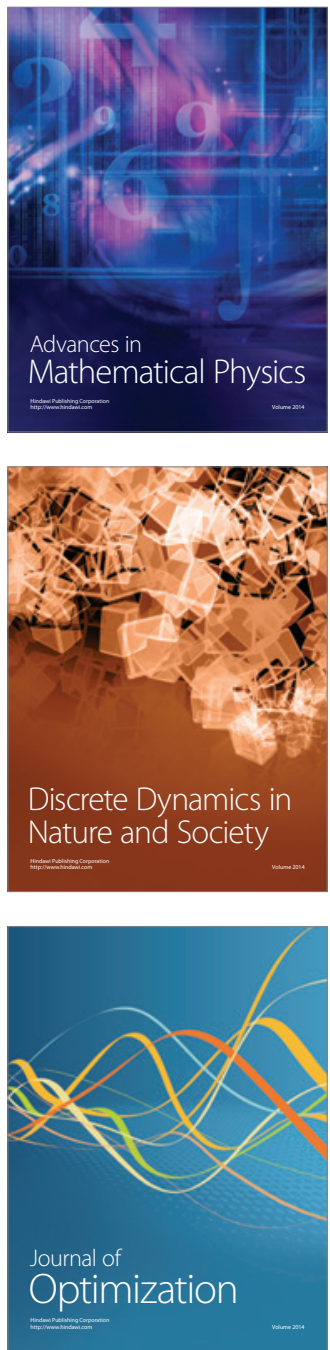